\title{
Option pricing under GARCH models applied to the SET50 index of Thailand
}

\author{
SOMPHORN ARUNSINGKARAT ${ }^{1}$, RENATO COSTA ${ }^{1}$, MASNITA MISRAN $^{2}$, \\ NATTAKORN PHEWCHEAN $* 1,3$ \\ ${ }^{1}$ Department of Mathematics, Faculty of Science, Mahidol University \\ Rama 6 rd., Bangkok 10400, THAILAND \\ ${ }^{2}$ School of Quantitative Sciences, UUM College of Arts and Sciences, Universiti Utara Malaysia, \\ 06010 UUM Sintok, Kedah Darul Aman, MALAYSIA. \\ ${ }^{3}$ Centre of Excellence in Mathematics, PERDO, THAILAND
}

\begin{abstract}
Variance changes over time and depends on historical data and previous variances; as a result, it is useful to use a GARCH process to model it. In this paper, we use the notion of Conditional Esscher transform to GARCH models to find the GARCH, EGARCH and GJR risk-neutral models. Subsequently, we apply these three models to obtain option prices for the Stock Exchange of Thailand and compare to the well-known Black-Scholes model. Findings suggest that most of the pricing options under GARCH model are the nearest to the actual prices for SET50 option contracts with both times to maturity of 30 days and 60 days.
\end{abstract}

Key-Words: - Option pricing, GARCH model, Stochastic assets.

Received: November 26, 2020. Revised: March 11, 2021. Accepted: March 28, 2021. Published: April 14, 2021.

\section{Introduction}

The most important options in Thailand are the SET50 index options which are the contracts that provide the right to the buyer to buy or sell the SET50 index on or before an expired date at a specified contract price, depending on the form of the option. We consider, in our study, the European call option and therefore it can only be exercised at expiration. Investment in the security has a risk due to changes over time and the variance may aff ect the investment returns in an unexpected way. The most popular model which gives the option price is the Black and Scholes formula which assumes volatility to be constant. Several studies have shown that in the market, the volatility of the security depends on previous volatilities. Hence the GARCH model is suitable for modeling volatility and for forecasting asset prices since the GARCH is a model that applies the information of variance in the past to forecast variances in the future.

Many researchers were interested in GARCH option pricing models. In 2010, Costa, Veiga and Siu [1] valuated option prices by using some special GARCH models based on risk neutral assumption. In 1995, Duan [2], developed an option pricing model and its corresponding delta formula for GARCH models with by the generalization of the concept of risk neutral valuation and local risk neutral valuation relationship on assets. In 1994, Gerber and Shiu [3] proposed a method of Esscher Transforms for option pricing which was later be able to apply for GARCH models. In the later year of 1996, Schmitt [4] showed his empirical studies that the time-varying volatility of asset returns can be described by GARCH models. Siu, Tong and Yang in 2004 [5] developed an approach for derivative pricing by using the GARCH option pricing models under the dynamic environment based on the model of Gerber-Shui and utilized the concrete idea of the conditional Esscher transforms. In 2017, Huang, Su and Chen [6] explored the valuation performance of a special kind of GARCH model, Heston and Nandi GARCH model on the option pricing. The results showed another vision of the impact of liquidity on GARCH models and the pricing error during financial crisis. In 2017, Badescu, Cui and Ortega [7] investigated the pricing and weak convergence of an asymmetric non-affine, non-Gaussian GARCH model. The option data analysis illustrated the advantage of coupling the option pricing with non-Gaussian methods. In 2018, Hua and Jiang [8] explored option pricing based on the proposed hybrid GARCH models with improved ensemble empirical mode decomposition. The results indicated that the hybrid models of GARCH with the decomposition technique could reduce the option pricing error. In the recent years, many researchers have applied GARCH models for the new financial currency which was well known as Cryptocurrency. In 2020, Venter, Mare and Pindza [9] applied GARCH models for Bitcoin and Cryptocurrency index (CRIX). Later, in the same year of 2020, 
Venter and Mare [10] applied GARCH models to generate volatility indices of Bitcoin and Cryptocurrency index (CRIX). In 2021, Anel, Rastegari and Stentoft [11] proposed a new condition of GARCH models for option pricing including a dynamic variance dependent pricing kernel.

There are some researchers studied GARCH models applied to the environment of Thailand. In 2005, Khanthavit [12] considered the model for pricing and analyzed the behavior of options on the SET50. His results show that the GARCH $(1,1)$ model could describe the random behavior of SET50 better than the other models. In 2014, Tanattrin [13] applied GARCH and GJRGARCH model for volatility analysis of international tourist arrival growth rate in Thailand.

In this paper, we study the GARCH, EGARCH and GJR-GARCH models to find the option prices. So we follow the Siu, Tong and Yang (2004) [5] method to find the risk neutral version of the GARCH, EGARCH and GJR-GARCH for pricing options on the SET50 index of Thailand.

\section{Theoretical Background}

In 1973, Black and Scholes [14] proposed a model for option pricing that has been frequently used in the financial researches. Nevertheless, the model that Black and Scholes proposed has some inapplicable assumptions of constant variance which is not practical. Hence, in 1982, Engle [15] presented the autoregressive conditional heteroskedasticity or $\mathrm{ARCH}$ model for modelling financial time series that present time-varying volatility clustering. In the ARCH model, a moving average of past error terms is utilized to forecast the variance:

$$
\sigma_{t}^{2}=\alpha_{0}+\alpha_{1} \xi_{t-1}^{2}+\ldots+\alpha_{q} \xi_{t-q}^{2}
$$

and $\xi_{t} \mid \phi_{t-1} \in N\left(0, \sigma_{t}^{2}\right)$ where $\alpha_{0}>0$ and $\alpha_{i}>0$, $i=1, \ldots, q . \alpha_{i}$ must be estimated from the given data, $\phi_{t}$ is the information set of all information until time $t$. The stationarity condition of the ARCH model is that $\sum_{i=1}^{q} \alpha_{i}<1$. The $\operatorname{GARCH}(p, q)$ model is well known as Generalized Autoregressive Conditionally Heteroskedastic with conditional variance with $p$ $\mathrm{GARCH}$ coefficient terms $\beta_{j}$ and $q \mathrm{ARCH}$ coefficient terms $\alpha_{i}$. It practically determines a time series of return $y_{t}=\mu+\xi_{t}$ where $\mu$ is the expected return and $\xi_{t}$ is a zero-mean white noise and

$$
\sigma_{t}^{2}=\alpha_{0}+\sum_{i=1}^{q} \alpha_{i} \xi_{t-i}^{2}+\sum_{j=1}^{p} \beta_{j} \sigma_{t-j}^{2}
$$

where $\xi_{t}=\sigma_{t} z_{t}$. The stationarity condition of the GARCH model is $\alpha_{0}>0, \alpha_{i} \geq 0, \quad \beta_{j} \geq 0$ and $\sum_{i=1}^{q} \alpha_{i}+\sum_{j=1}^{p} \beta_{j}<1$

In 1991, Nelson [16] presented the exponential GARCH or EGARCH model in which the logarithm of conditional variance is as follows.

$$
\begin{gathered}
\log \sigma_{t}^{2}=\alpha_{0}+\sum_{i=1}^{q} \alpha_{i}\left\lfloor\frac{\left|\xi_{t-i}\right|}{\sigma_{t-i}}-E\left(\frac{\left|\xi_{t-i}\right|}{\sigma_{t-i}}\right)\right\rfloor \\
+\sum_{j=1}^{p} \beta_{j} \log \sigma_{t-j}^{2}+\sum_{i=1}^{q} \gamma\left\lfloor\frac{\xi_{t-i}}{\sigma_{t-i}}\right\rfloor
\end{gathered}
$$

where $\xi_{t} \in N\left(0, \sigma_{t}^{2}\right)$.

In the GJR model, $I\left[\xi_{t-i}<0\right]=1$ or $I\left[\xi_{t-i} \geq 0\right]=0$ where $\alpha_{0}>0, \alpha_{i} \geq 0, \beta_{j} \geq 0$ and $\alpha_{i}+\xi_{i} \geq 0$.

The stationarity condition of the GJR model is:

$$
\sum_{i=1}^{q} \alpha_{i}+\sum_{j=1}^{p} \beta_{j}+\frac{1}{2} \sum_{i=1}^{q} \gamma_{i}<1
$$

In this paper, we consider the case of the GARCH, EGARCH and GJRGARCH option pricing models with the assumptions of the conditional distribution of the innovations $\xi_{t}$ given that $\phi_{t-1}$ is normal.

\subsection{GARCH Option Pricing Model}

For GARCH option pricing model, it is provided that $\varepsilon_{t}$, given $\phi_{t-1}$, is a noise with conditional mean zero and variance $\sigma_{t}^{2}$ under the statistical probability measure $P$. From the work of Duan in 1995 [2], we suppose that the process $\left\{\xi_{t}\right\}_{t \in T}$ can be modelled as a $\operatorname{GARCH}(p, q)$ with zero mean and variance $\sigma_{t}^{2}$ under the probability $P$.

Thus, we have that $Y_{t}$ under the probability measure $P$ is distributed under the normal distribution with the mean of $r+\lambda \sqrt{\sigma_{t}^{2}}-\sigma_{t}^{2} / 2$ and the variance of $\sigma_{t}^{2}$. The conditional risk-neutralized Esscher parameter $\theta_{t}^{q}$ is given by

$$
r=\ln \left\{M_{Y}\left(t, \theta_{t}^{q}+1\right)\right\} \text {. }
$$

Using

$$
\theta_{t}^{q}=\frac{r-\left(\mu+\left(\sigma_{t}^{2} / 2\right)\right)}{\sigma_{t}^{2}}
$$

we have

$$
M_{Y}^{\theta}\left(t, z+\theta_{t}^{q}\right)=\frac{M_{Y}\left(t, z+\theta_{t}^{q}\right)}{M_{Y}\left(t, \theta_{t}^{q}\right)}=e^{z\left(r-\sigma_{t}^{2} / 2+z^{2} \sigma_{t}^{2} / 2\right)}
$$


which is a normally distributed moment generating function with mean $r-\sigma_{t}^{2} / 2$ and variance $\sigma_{t}^{2}$, with

$$
Y_{t}^{\theta} \square N\left(r-\sigma_{t}^{2} / 2, \sigma_{t}^{2} / 2\right) .
$$

Hence, the conditional distribution of $\xi_{t}$ given $\phi_{t-1}$ under $P$ where

is

$$
Y_{t}=\mu+\varepsilon_{t} ; \xi_{t}=\varepsilon_{t} \sqrt{\sigma_{t}^{2}}
$$

$$
\begin{gathered}
E_{P}\left[\xi_{t} \mid \phi_{t-1}\right]=E_{P}\left[Y_{t} \mid \phi_{t}\right]-\mu=r-\frac{\sigma_{t}^{2}}{2}-\mu \\
\operatorname{Var}_{P}\left[\xi_{t} \mid \phi_{t-1}\right]=\operatorname{Var}_{P}\left[Y_{t}\right]=\sigma_{t}^{2} .
\end{gathered}
$$

Under $Q$, nevertheless, the conditional distribution of $\xi_{t}$ given $\phi_{t-1}$ is $N\left(0, \sigma_{t}^{2}\right)$,

and

$$
\varepsilon_{t}=\xi_{t}-r+\mu+\sigma_{t}^{2} / 2
$$

$$
Y_{t}=r-\sigma_{t}^{2} / 2+\varepsilon_{t} .
$$

It is noted that by applying the conditional mean, $\mu=r+\lambda \sqrt{\sigma_{t}^{2}}-\sigma_{t}^{2} / 2$, the same way as in Duan's work [2], we obtain

$$
\varepsilon_{t}=\xi_{t}-r+r+\lambda \sqrt{\sigma_{t}^{2}}-\sigma_{t}^{2} / 2+\sigma_{t}^{2} / 2 .
$$

Thus,

$$
\xi_{t}=\varepsilon_{t}-\lambda \sqrt{\sigma_{t}^{2}}
$$

and therefore the dynamics of the variance is given by

$$
\sigma_{t}^{2}=\alpha_{0}+\sum_{i=1}^{P} \alpha_{i}\left(\varepsilon_{t}-\lambda \sqrt{\sigma_{t-1}^{2}}\right)^{2}+\sum_{j=1}^{q} \beta_{j} \sigma_{t-j}^{2} .
$$

This result was obtained from Siu, Tong and Yang [5] and was in agreement with that obtained by Duan [2].

\subsection{EGARCH Option Pricing Model}

The EGARCH $(1,1)$ model under the statistical probability measure $P$ is given by:

$$
\log \sigma_{t}^{2}=\alpha_{0}+\alpha\left[\frac{\left|\xi_{t-1}\right|}{\sigma_{t-1}}-\sqrt{\frac{2}{\pi}}\right]+\beta \log \sigma_{t-1}^{2}+\gamma\left[\frac{\xi_{t-1}}{\sigma_{t-1}}\right] .
$$

In a similar manner as in the previous case, we apply the method used by Sui, Tong and Yang in [5] to obtain the EGARCH under the $Q$ measure:

$$
\begin{gathered}
\log \sigma_{t}^{2}=\alpha_{0}+\alpha\left[\frac{\left|\varepsilon_{t-1}-\lambda \sqrt{\sigma_{t-1}^{2}}\right|}{\sigma_{t-1}}-\sqrt{\frac{2}{\pi}}\right] \\
+\beta \log \sigma_{t-1}^{2}+\gamma\left\lfloor\frac{\varepsilon_{t-1}-\lambda \sqrt{\sigma_{t-1}^{2}}}{\sigma_{t-1}}\right\rfloor .
\end{gathered}
$$

\subsection{GJR-GARCH Option Pricing Model}

The GJR $(1,1)$ model under the statistical probability measure $P$ is given by :

$$
\sigma_{t}^{2}=\alpha_{0}+\alpha \xi_{t-1}^{2}+\beta \sigma_{t-1}^{2}+\lambda I\left[\xi_{t-1}<0\right] \xi_{t-1}^{2} .
$$

In a similar manner as in the previous cases, we apply the method used by Sui, Tong and Yang in [5]. Now, we have the GJR-GARCH under $Q$ measure as follows:

$$
\begin{gathered}
\sigma_{t}^{2}=\alpha_{0}+\alpha\left(\varepsilon_{t}-\lambda \sqrt{\sigma_{t}^{2}}\right)^{2}+\beta \sigma_{t-1}^{2} \\
+\gamma I\left[\varepsilon_{t}<\lambda \sqrt{\sigma_{t}^{2}}\right]\left(\varepsilon_{t}-\lambda \sqrt{\sigma_{t}^{2}}\right)^{2} .
\end{gathered}
$$

\section{Methodology}

In this work, we make some assumptions to construct a financial model for the discrete environment. We let $T$ be the set $\{0,1,2, \ldots, T\}$ representing economic time under the probability space $(\Omega, F, P)$ where $P$ is the probability measure with sample space $\Omega$ and event space $F$.

Let $\xi=\left\{\xi_{t}\right\}_{t \in T}$ be a stochastic process of underlying asset return and $\sigma_{t}^{2}$ denotes the stock variance. Next, we let $\lambda$ be the risk premium and $r$ be the risk free rate. Under $P$, we presume that the process $\left\{\beta_{t}\right\}_{t \in T}$, the bond price process, follows the relation $\beta_{t}=\beta_{t-1} e^{r}, \beta_{0}=1$ and the process of stock price $\left\{S_{t}\right\}_{t \in T}$ follows the dynamics,

$$
\begin{gathered}
Y_{t}=r+\lambda \sqrt{\sigma_{t}^{2}}-\frac{1}{2} \sigma_{t}^{2}+\varepsilon_{t}, \quad S_{0}=S, \\
\varepsilon_{t} \square N\left(0, \sigma_{t}^{2}\right), \quad t \in T-\{0\}
\end{gathered}
$$

where $Y_{t}=\ln \frac{S_{t}}{S_{t-1}}$ denotes the log-return of the stock $S$ [2]. Subsequently, the method of conditional Esscher transform is applied for $Y_{t}$, the GARCH process with conditional Esscher parameter sequence of $\left\{\theta_{t}\right\}_{t \in T}$.

Under the probability measure $P$, we let $M_{Y_{t} \mid \phi_{t-1}}(z)$ be the moment generating function of $Y_{t}$ given the information $\phi_{t-1}$ at time $t-1$ where $z \in \square$ and assume a stochastic process $\left\{\theta_{t}\right\}_{t \in T-\{0\}}$ given the information $\phi_{t-1}$. We have

$$
\begin{aligned}
& M_{Y_{t}}(t, z):=E_{P}\left(e^{z Y_{t}} \mid \phi_{t-1}\right) \\
& =\int_{-\infty}^{\infty} e^{z Y_{t}} d F\left(y \mid r+\lambda \sqrt{\sigma_{t}^{2}}-\frac{1}{2} \sigma_{t}^{2}+\varepsilon_{t}, \sigma_{t}^{2}\right)
\end{aligned}
$$

where a conditional distribution function of $Y_{t}$ is defined as 


$$
F\left(y \mid r+\lambda \sqrt{\sigma_{t}^{2}}-\frac{1}{2} \sigma_{t}^{2}+\varepsilon_{t}, \sigma_{t}^{2}\right)=P\left(Y_{t} \leq \phi_{t-1}\right) .
$$

We assume that, for each $t \in T-\{0\}$ and $z \in \square$, $M_{Y_{t}}(t, z)$ exists if $E_{P}\left(e^{z Y_{t}} \mid \phi_{t-1}\right)<\infty$ and $M_{Y_{t}}(t, \theta)$ exists and define $\Lambda_{0}=1$, where $E$ is the expectation under $P$ and

$\Lambda_{t}=\prod_{k=1}^{t} \frac{e^{\theta_{k} Y_{k}}}{M_{Y}\left(k, \theta_{k}\right)}, \quad t \in T-\{0\}$.

Then, we let $\left\{P_{t, \Lambda_{t}}\right\}_{t \in T-\{0\}}$ be a family of probabilities and define

$$
\begin{aligned}
& P_{t, \Lambda_{t}}\left(\left\{Y_{t} \in X\right\} \mid \phi_{t-1}\right) \\
& =E_{p_{t}}\left(I\left(Y_{t} \in X\right) \frac{e^{\theta_{t} Y_{t}}}{E_{P_{t}}\left(e^{\theta_{t} Y_{t}} \mid \phi_{t-1}\right)} \mid \phi_{t-1}\right)
\end{aligned}
$$

where $I\left\{Y_{t} \in X\right\}$ is the indicator function of the event and $X$ is an open set of the real line. Letting $M_{Y}^{\theta}\left(z, \theta_{t}\right)$ be the moment generating function of the return $Y_{t}$ given the information $\phi_{t-1}$ with the conditional Esscher parameter $\phi_{t}$, we consequently have

$$
M_{Y}^{\theta}\left(z, \theta_{t}\right)=\frac{M_{Y}\left(t, z+\theta_{t}\right)}{M_{Y}\left(t, \theta_{t}\right)}
$$

With a sequence of $\left\{\theta_{t}^{q}\right\}_{t \in T-\{0\}}$, we assume that log-return follows the following equation with the martingale condition.

$$
r=\ln \left\{M_{Y}\left(t, \theta_{t}^{q}+1\right)\right\}-\ln \left\{M_{Y}\left(t, \theta_{t}^{q}\right)\right\}, \quad t \in T-\{0\}
$$

In 1994, Shiu et al [3] proposed the derivative pricing model $V$ at time $t \in T$ as

$V_{t}=e^{-r(T-t)} E_{Q}\left(V_{T} \mid \phi_{t}\right)$

where $Q$ is a conditional risk neutral Esscher pricing measure and $P_{T, \Lambda_{T}^{q}}$ is a probability measure on $F=\phi_{T}$. The process $\left\{e^{-r t} S_{t}\right\}_{t \in T}$ is a martingale process under $Q$ given the information $\phi$.

Consequently, in this paper, the methodology is performed in the following steps.

1. We randomly choose the option contract on SET50 market of Thailand from years 2015 and 2016, since the data of these two years are publicly accessible with complete data.

2. We perform the residual test of ARCH effect from the chosen option data fitness by using LjungBox's $Q^{2}(n)$ test. The results of $Q(20)$ of 25.382 and $Q^{2}(20)$ of 52.320 show that SET50 option price samples have the ARCH effect.
3. In this paper, we use the lag order $(1,1)$ for all testing models, since it was shown that models with a small lag are sufficient to cope with the changing variance [17].

4. We utilize the maximum likelihood estimation for parameter estimates of GARCH, EGARCH and GJR-GARCH.

5. From equation (6), the expected value of $V_{T}$ is obtained by Monte Carlo simulation for a sample size of 10,000 with the variance reduction technique which is a control variate method.

6 . The option pricing value from classical BlackSholes model is obtained from a financial toolbox of Matlab.

7. The method of room mean square error is applied to evaluate the precision performance of the GARCH models.

8. We discuss the advantage and the limitation of the results for the investor in stock market of Thailand.

\section{Numerical Results}

In this section, we show some simulated option prices by using GARCH option pricing models (GARCH, EGARCH and GJR) and the classical Black-Scholes model (BS) under probability measure $Q$ using the estimated values of SET50. The data have been obtained from the database www.set.or.th and the derivative security in which we are interested is the European Call option on the SET50 index.

We randomly pick the option contract on SET50 market from years 2015 and 2016. The contracts in year 2015 under study are S50Z15C950, S50Z15C925, S50Z15C900, S50Z15C875, S50Z15C850, S50Z15P950, S50Z15P925, S50Z15P900, S50Z15P875 and S50Z15P850. And in year 2016, the contracts under study are S50Z16C1000, S50Z16C975, S50Z16C950, S50Z16C925, S50Z16C900, S50Z16P1000, S50Z16P975, S50Z16P950, S50Z16P925 and S50Z16P900. We use daily closing prices in each index to estimate the parameters of the GARCH, EGARCH and GJR-GARCH models to be able to find the option price. 
Table 1 The Estimated Parameters of the GARCH models for contracts year 2015

\begin{tabular}{|c|c|c|c|c|c|}
\hline \multirow{2}{*}{ Contract } & \multirow{2}{*}{ Models } & \multicolumn{4}{|c|}{ Parameters } \\
\hline & & $\alpha_{0}$ & $\alpha$ & $\beta$ & $\gamma$ \\
\hline \multirow{3}{*}{ S50Z15C950 } & GARCH & $1.79 e-5$ & 0.92810 & 0.99183 & - \\
\hline & EGARCH & -6.29874 & 0.12032 & -0.79121 & -0.12890 \\
\hline & GJR & $2.37 \mathrm{e}-5$ & 0.89321 & 0.21083 & 0.13801 \\
\hline \multirow{3}{*}{ S50Z15C925 } & GARCH & $2.28 \mathrm{e}-5$ & 0.23018 & 0.64020 & - \\
\hline & EGARCH & -3.39023 & -0.11930 & 0.20188 & -0.13284 \\
\hline & GJR & $2.11 \mathrm{e}-5$ & 0.20912 & 0.80239 & 0.11121 \\
\hline \multirow{3}{*}{ S50Z15C900 } & GARCH & $2.01 \mathrm{e}-5$ & 0.39012 & 0.53082 & - \\
\hline & EGARCH & -1.39801 & -0.00231 & 0.98210 & -0.19021 \\
\hline & GJR & $2.55 \mathrm{e}-5$ & 0.32900 & 0.99821 & 0.11934 \\
\hline \multirow{3}{*}{ S50Z15C875 } & GARCH & $2.83 \mathrm{e}-5$ & 0.12903 & 0.10299 & - \\
\hline & EGARCH & -0.49781 & -0.02190 & -0.28301 & -0.12190 \\
\hline & GJR & $2.29 \mathrm{e}-5$ & 0.23188 & 0.90128 & 0.13891 \\
\hline \multirow{3}{*}{$\mathrm{S} 50 \mathrm{Z} 15 \mathrm{C} 850$} & GARCH & $1.88 \mathrm{e}-5$ & 0.11408 & 0.22180 & - \\
\hline & EGARCH & -10.81006 & 0.93801 & 0.90721 & -0.17781 \\
\hline & GJR & $2.17 \mathrm{e}-5$ & 0.15324 & 0.28102 & 0.11198 \\
\hline \multirow{3}{*}{ S50Z15P950 } & GARCH & $3.74 \mathrm{e}-5$ & 0.32301 & 0.92108 & - \\
\hline & EGARCH & -2.89102 & -0.00513 & -0.27912 & -0.17782 \\
\hline & GJR & $2.02 \mathrm{e}-5$ & 0.34782 & 0.82109 & 0.11293 \\
\hline \multirow{3}{*}{ S50Z15P925 } & GARCH & $3.25 \mathrm{e}-5$ & 0.01812 & 0.80122 & - \\
\hline & EGARCH & -5.90123 & -0.09913 & 0.21092 & -0.18329 \\
\hline & GJR & $2.65 e-5$ & 0.05635 & 0.99218 & 0.13245 \\
\hline \multirow{3}{*}{ S50Z15P900 } & GARCH & $3.71 \mathrm{e}-5$ & 0.52313 & 0.71221 & - \\
\hline & EGARCH & -0.44814 & 0.02921 & 0.82123 & -0.19810 \\
\hline & GJR & $2.62 \mathrm{e}-5$ & 0.43872 & 0.39033 & 0.17812 \\
\hline \multirow{3}{*}{ S50Z15P875 } & GARCH & $2.90 \mathrm{e}-5$ & 0.62901 & 0.59023 & - \\
\hline & EGARCH & -8.11356 & -0.13792 & 0.60298 & -0.18921 \\
\hline & GJR & $2.07 \mathrm{e}-5$ & 0.58729 & 0.21203 & 0.12782 \\
\hline \multirow{3}{*}{ S50Z15P850 } & GARCH & $1.66 \mathrm{e}-5$ & 0.80001 & 0.12324 & - \\
\hline & EGARCH & -11.18182 & -0.98921 & -0.83232 & -0.16692 \\
\hline & GJR & $2.35 \mathrm{e}-5$ & 0.78293 & 0.79231 & 0.19320 \\
\hline
\end{tabular}

Table 1 and 2 shows estimation results for randomized contracts from year 2015 and 2016 respectively, based on the same index. Several researchers assume the risk-free rate $r$ to be 0 to make the interpretation of options easier such as Duan in 1995 [3] and Schmitt in 1996 [4]. Consequently, in this work, we also assume that the risk premium is zero.

Next, we use the estimated parameters from Table 1 and 2 in GARCH models under probability measure $Q$ so that we find the option price by taking the conditional expectation of the terminal payoff under the pricing probability measure $Q$ and discount at the risk-free interest rate as follows:

$$
C_{t}=e^{-r(T-t)} E\left[\max \left(S_{T}-X, 0\right)\right],
$$

and

$$
P_{t}=e^{-r(T-t)} E\left[\max \left(0, X-S_{T}\right)\right]
$$

where $C_{t}$ and $P_{t}$ are call option prices and put option prices respectively. Following the work of Duan's in 1995 [2], the terminal stock price at time $T$ can be calculated as:

$$
S_{T}=S_{t} \exp \left[(T-t) r-\frac{1}{2} \sum_{s=t+1}^{T} \sigma_{s}^{2}+\sum_{s=t+1}^{T} \xi_{s}\right] .
$$

Tables 3 and 4 present the average simulated option prices $C_{t}$ and $P_{t}$ under GARCH, EGARCH and GJRGARCH models applied to the SET50 index option. This table uses the estimated parameters from Tables 1 and 2 to fit in the GARCH, EGARCH and GJRGARCH option pricing models. Then, we establish a set of parameters, the strike price $(K)$ and stock price $\left(S_{0}\right)$ at the start date of the year-2015 contracts and year-2016 contracts which are 19 August 2015 and 2 November 2016 for calculation, 
Table 2 The Estimated Parameters of the GARCH models for contracts year 2016

\begin{tabular}{|l|l|c|c|c|c|}
\hline \multirow{2}{*}{ Contract } & \multirow{2}{*}{ Models } & \multicolumn{4}{|c|}{ Parameters } \\
\cline { 2 - 6 } & & $\alpha_{0}$ & $\alpha$ & $\beta$ & $\gamma$ \\
\hline \multirow{3}{*}{ S50Z16C1000 } & GARCH & $1.63 \mathrm{e}-5$ & 0.42532 & 0.41512 & - \\
\cline { 2 - 6 } & EGARCH & -6.34532 & 0.25104 & -0.89412 & -0.15343 \\
\cline { 2 - 6 } & GJR & $3.52 \mathrm{e}-5$ & 0.23482 & 0.24255 & 0.13241 \\
\hline \multirow{3}{*}{ S50Z15C975 } & GARCH & $1.58 \mathrm{e}-5$ & 0.57923 & 0.84152 & - \\
\cline { 2 - 6 } & EGARCH & -4.32144 & 0.94210 & 0.25143 & -0.19423 \\
\cline { 2 - 6 } & GJR & $2.72 \mathrm{e}-5$ & 0.45921 & 0.74231 & 0.18894 \\
\hline \multirow{3}{*}{ S50Z15C925 } & GARCH & $2.32 \mathrm{e}-5$ & 0.41325 & 0.42398 & - \\
\cline { 2 - 6 } & EGARCH & -1.43253 & -0.04221 & 0.74235 & -0.11048 \\
\cline { 2 - 6 } & GJR & $4.23 \mathrm{e}-5$ & 0.39802 & 0.74632 & 0.18432 \\
\hline & GARCH & $2.48 \mathrm{e}-5$ & 0.24234 & 0.67333 & - \\
\cline { 2 - 6 } & GGARCH & -0.23642 & 0.43152 & -0.70244 & -0.18923 \\
\cline { 2 - 6 } & GJR & $3.54 \mathrm{e}-5$ & 0.28391 & 0.78234 & 0.12294 \\
\hline & GARCH & $2.09 \mathrm{e}-5$ & 0.21425 & 0.23249 & - \\
\cline { 2 - 6 } & EGARCH & -3.42220 & 0.43232 & 0.74125 & -0.14214 \\
\cline { 2 - 6 } & GJR & $4.88 \mathrm{e}-5$ & 0.23008 & 0.94325 & 0.10943 \\
\hline \multirow{3}{*}{ S50Z15P1000 } & GARCH & $2.23 \mathrm{e}-5$ & 0.24202 & 0.24244 & - \\
\cline { 2 - 6 } & GGARCH & -5.23521 & -0.09323 & -0.94125 & -0.10044 \\
\cline { 2 - 6 } & GJR & $2.56 \mathrm{e}-5$ & 0.22329 & 0.73221 & 0.17935 \\
\hline \multirow{3}{*}{ S50Z15P975 } & GARCH & $2.49 \mathrm{e}-5$ & 0.04323 & 0.73242 & - \\
\cline { 2 - 6 } & EGARCH & -1.94233 & -0.03253 & 0.12532 & -0.17354 \\
\cline { 2 - 6 } & GJR & $3.92 \mathrm{e}-5$ & 0.03928 & 0.81435 & 0.19843 \\
\hline \multirow{3}{*}{ S50Z15P950 } & GARCH & $2.47 \mathrm{e}-5$ & 0.28423 & 0.54523 & - \\
\cline { 2 - 6 } & EGARCH & -0.32424 & -0.04238 & 0.82415 & -0.19432 \\
\cline { 2 - 6 } & GJR & $1.34 \mathrm{e}-5$ & 0.23654 & 0.43202 & 0.15324 \\
\hline & GARCH & $2.76 \mathrm{e}-5$ & 0.69023 & 0.42150 & - \\
\cline { 2 - 6 } & EGARCH & -5.10023 & -0.13225 & 0.84215 & -0.17842 \\
\cline { 2 - 6 } & GJR & $3.34 \mathrm{e}-5$ & 0.69832 & 0.23114 & 0.11894 \\
\cline { 2 - 6 } & GARCH & $1.91 \mathrm{e}-5$ & 0.34221 & 0.74231 & -0.28340 \\
\cline { 2 - 6 } & EGARCH & -12.43253 & -0.04247 & -0.88349 & 0.29306 \\
\hline & GJR & $2.92 \mathrm{e}-5$ & 0.42983 & 0.32566 & \\
\hline & & & & - \\
\hline
\end{tabular}

respectively. The values of parameters set in the models of each contract are clearly shown in Table 5. Thus, we apply the diff erences between the actual data of option prices and the simulated results in order to compare the performance of the models.

Tables 6 and 7 show the Root Mean Square Error (or RMSE), calculated by the square root of average square diff erence between the simulated option price and the option price in the market. The formula of RMSE is as follows:

$$
R M S E=\sqrt{\frac{1}{n} \sum_{i=1}^{n}\left(\hat{y}_{i}-y_{i}\right)^{2}}
$$

where $\hat{y}_{i}$ are predicted prices, and $y_{i}$ market prices. We simulated the prices of option under the GARCH, EGARCH and GJRGARCH models by using MATLAB. The contracts under study from year 2015 are S50Z15C950, S50Z15C925, S50Z15C900, S50Z15C875, S50Z15C850, S50Z15P950,
S50Z15P925, S50Z15P900, S50Z15P875 and S50Z15P850.

The results show that, at the maturity time of 30 days, the GARCH model's value is the nearest to the actual option price in the market in all of our sample contracts except that the option prices for the contract S50Z15C850 under the EGARCH model are slightly nearer to the actual option prices as it can be seen that RMSE of contract S50Z15C850 under GARCH model is 0.17189 and the RMSE of EGARCH model is 0.16727 .

At maturity time of 60 days, the option prices under the GARCH model are the nearest to the actual option prices in the market for all of the contracts as well, except that the for the contract of S50Z15C950, the EGARCH model shows a slightly better performance as indicated by the results that the RMSE of contract S50Z15C950 with GARCH model is 0.01332 , while it is 0.01325 with the EGARCH. 
WSEAS TRANSACTIONS ON MATHEMATICS DOI: 10.37394/23206.2021.20.112
Somphorn Arunsingkarat, Renato Costa,

Masnita Misran, Nattakorn Phewchean
Table 3 The average simulated option prices under GARCH and BS models for contracts year 2015

\begin{tabular}{|l|c|c|c|c|}
\hline Contract & GARCH & EGARCH & GJR & BS \\
\hline Call option & \multicolumn{5}{|c|}{$T=30$} \\
\hline S50Z15C950 & 2.5823 & 3.2409 & 11.6788 & 9.7283 \\
\hline S50Z15C925 & 6.3679 & 7.9823 & 12.8396 & 11.2452 \\
\hline S50Z15C900 & 11.5321 & 14.0032 & 16.8921 & 17.3340 \\
\hline S50Z15C875 & 24.0002 & 25.3245 & 29.9892 & 32.2391 \\
\hline S50Z15C850 & 34.8772 & 34.7458 & 35.1242 & 40.9811 \\
\hline Put option & \multicolumn{5}{|c|}{$T=30$} \\
\hline S50Z15P950 & 63.5023 & 63.4903 & 68.5322 & 65.0927 \\
\hline S50Z15P925 & 46.1098 & 46.5436 & 58.3423 & 56.3902 \\
\hline S50Z15P900 & 25.7320 & 27.3432 & 28.0321 & 35.0924 \\
\hline S50Z15P875 & 14.2232 & 16.9823 & 19.9302 & 25.2501 \\
\hline S50Z15P850 & 8.20102 & 11.2422 & 17.4325 & 20.2239 \\
\hline Call option & \multicolumn{5}{|c|}{$T=60$} \\
\hline S50Z15C950 & 3.4245 & 3.3129 & 17.3274 & 15.3022 \\
\hline S50Z15C925 & 7.5241 & 7.6432 & 35.4325 & 26.3247 \\
\hline S50Z15C900 & 12.353 & 12.453 & 50.4522 & 49.2932 \\
\hline S50Z15C875 & 26.432 & 29.432 & 70.0432 & 72.4320 \\
\hline S50Z15C850 & 36.534 & 40.024 & 80.3245 & 93.7882 \\
\hline Put option & 66.2932 & 66.4565 & 140.0623 & 80.3202 \\
\hline S50Z15P950 & 69.4324 & 49.5343 & 75.9023 & 69.3936 \\
\hline S50Z15P925 & 48.4324 & 49.9457 \\
\hline S50Z15P900 & 27.0801 & 28.4326 & 47.0343 & 38.9021 \\
\hline S50Z15P875 & 17.0023 & 20.0342 & 36.0325 & 29.0287 \\
\hline S50Z15P850 & 11.8425 & 15.0425 & 24.3883 & \\
\hline
\end{tabular}

Table 4 The average simulated option prices under GARCH and BS models for contracts year 2016

\begin{tabular}{|l|c|c|c|c|}
\hline Contract & GARCH & EGARCH & GJR & BS \\
\hline Call option & \multicolumn{5}{|c|}{$T=30$} \\
\hline S50Z16C1000 & 7.24254 & 7.13254 & 9.42423 & 10.1324 \\
\hline S50Z16C975 & 15.42344 & 15.04353 & 18.23494 & 19.9932 \\
\hline S50Z16C950 & 26.42435 & 26.14252 & 31.32544 & 35.3211 \\
\hline S50Z16C925 & 41.12325 & 41.04659 & 42.33256 & 53.2038 \\
\hline S50Z16C900 & 55.62234 & 58.12524 & 61.04235 & 73.9217 \\
\hline Put option & \multicolumn{5}{|c|}{$T=30$} \\
\hline S50Z16P1000 & 67.83224 & 67.18942 & 75.45336 & 78.3231 \\
\hline S50Z16P975 & 47.32532 & 47.10425 & 49.23343 & 52.3292 \\
\hline S50Z16P950 & 32.32556 & 31.54342 & 31.24797 & 43.4893 \\
\hline S50Z16P925 & 22.22145 & 24.52345 & 30.09842 & 35.4581 \\
\hline S50Z16P900 & 14.23223 & 16.52533 & 29.04253 & 29.9210 \\
\hline Call option & \multicolumn{5}{|c|}{$T=60$} \\
\hline S50Z16C1000 & 9.45233 & 10.42352 & 11.42026 & 12.9332 \\
\hline S50Z16C975 & 18.23123 & 18.34235 & 23.89421 & 19.3292 \\
\hline S50Z16C950 & 28.24252 & 30.24235 & 37.42363 & 35.9844 \\
\hline S50Z16C925 & 43.43235 & 45.25324 & 46.42546 & 56.2001 \\
\hline S50Z16C900 & 58.42224 & 61.02644 & 59.42084 & 65.7862 \\
\hline Put option & \multicolumn{5}{|c|}{$T=60$} \\
\hline S50Z16P1000 & 69.42352 & 69.94326 & 75.42634 & 78.4522 \\
\hline S50Z16P975 & 50.23425 & 51.14253 & 53.24336 & 65.0901 \\
\hline S50Z16P950 & 34.53426 & 36.00234 & 43.23509 & 54.2995 \\
\hline S50Z16P925 & 23.32098 & 24.14253 & 30.42540 & 41.7890 \\
\hline S50Z16P900 & 15.94204 & 17.45530 & 25.42543 & 30.0087 \\
\hline
\end{tabular}

Table 5 Parameters set in the models for contracts

\begin{tabular}{|c|c|c|c|c|}
\hline Contract & $K$ & $S_{0}$ & Variance & $\begin{array}{c}\text { Actual } \\
\text { option } \\
\text { price }\end{array}$ \\
\hline Call option & \multicolumn{4}{|c|}{$T=30$} \\
\hline S50Z15C950 & 950 & 859.51 & 0.234523 & 2.90 \\
\hline S50Z15C925 & 925 & 859.51 & 0.134425 & 6.00 \\
\hline S50Z15C900 & 900 & 859.51 & 0.135234 & 11.80 \\
\hline S50Z15C875 & 875 & 859.51 & 0.342543 & 24.00 \\
\hline S50Z15C850 & 850 & 859.51 & 0.109452 & 34.50 \\
\hline Put option & \multicolumn{4}{|c|}{$T=30$} \\
\hline S50Z15P950 & 950 & 859.51 & 0.123253 & 63.80 \\
\hline S50Z15P925 & 925 & 859.51 & 0.143567 & 46.00 \\
\hline S50Z15P900 & 900 & 859.51 & 0.193235 & 25.90 \\
\hline S50Z15P875 & 875 & 859.51 & 0.289223 & 14.30 \\
\hline S50Z15P850 & 850 & 859.51 & 0.242674 & 8.80 \\
\hline Call option & \multicolumn{4}{|c|}{$T=30$} \\
\hline S50Z16C1000 & 1000 & 942.56 & 0.192523 & 7.90 \\
\hline S50Z16C975 & 975 & 942.56 & 0.145437 & 15.70 \\
\hline S50Z16C950 & 950 & 942.56 & 0.183257 & 26.60 \\
\hline S50Z16C925 & 925 & 942.56 & 0.235367 & 40.40 \\
\hline S50Z16C900 & 900 & 942.56 & 0.323426 & 56.00 \\
\hline Put option & \multicolumn{4}{|c|}{$T=30$} \\
\hline S50Z16P1000 & 1000 & 942.56 & 0.132567 & 67.90 \\
\hline S50Z16P975 & 975 & 942.56 & 0.282363 & 48.00 \\
\hline S50Z16P950 & 950 & 942.56 & 0.427336 & 32.30 \\
\hline S50Z16P925 & 925 & 942.56 & 0.291325 & 21.30 \\
\hline S50Z16P900 & 900 & 942.56 & 0.225623 & 13.90 \\
\hline Call option & \multicolumn{4}{|c|}{$T=60$} \\
\hline S50Z15C950 & 950 & 859.51 & 0.242536 & 3.60 \\
\hline S50Z15C925 & 925 & 859.51 & 0.292677 & 7.00 \\
\hline S50Z15C900 & 900 & 859.51 & 0.192364 & 12.60 \\
\hline S50Z15C875 & 875 & 859.51 & 0.242673 & 25.20 \\
\hline S50Z15C850 & 850 & 859.51 & 0.109452 & 36.00 \\
\hline Put option & \multicolumn{4}{|c|}{$T=60$} \\
\hline S50Z15P950 & 950 & 859.51 & 0.224256 & 64.60 \\
\hline S50Z15P925 & 925 & 859.51 & 0.142267 & 48.00 \\
\hline S50Z15P900 & 900 & 859.51 & 0.122566 & 27.90 \\
\hline S50Z15P875 & 875 & 859.51 & 0.133554 & 15.30 \\
\hline S50Z15P850 & 850 & 859.51 & 0.272342 & 10.80 \\
\hline Call option & \multicolumn{4}{|c|}{$T=60$} \\
\hline S50Z16C1000 & 1000 & 942.56 & 0.152623 & 9.90 \\
\hline S50Z16C975 & 975 & 942.56 & 0.183265 & 16.50 \\
\hline S50Z16C950 & 950 & 942.56 & 0.223563 & 28.20 \\
\hline S50Z16C925 & 925 & 942.56 & 0.243003 & 42.50 \\
\hline S50Z16C900 & 900 & 942.56 & 0.132578 & 58.00 \\
\hline Put option & \multicolumn{4}{|c|}{$T=60$} \\
\hline S50Z16P1000 & 1000 & 942.56 & 0.225367 & 69.50 \\
\hline S50Z16P975 & 975 & 942.56 & 0.242774 & 49.00 \\
\hline S50Z16P950 & 950 & 942.56 & 0.243268 & 34.00 \\
\hline S50Z16P925 & 925 & 942.56 & 0.112532 & 23.20 \\
\hline S50Z16P900 & 900 & 942.56 & 0.173564 & 15.10 \\
\hline
\end{tabular}


Table 6 Model comparison for 2015

contracts.

\begin{tabular}{|c|c|c|c|c|}
\hline Contract & GARCH & EGARCH & GJR & BS \\
\hline Call option & \multicolumn{5}{|c|}{$T=30$} \\
\hline S50Z15C950 & 0.1982 & 1.4823 & 3.5802 & 3.4293 \\
\hline S50Z15C925 & 0.2452 & 1.4562 & 2.2063 & 2.1293 \\
\hline S50Z15C900 & 0.2639 & 1.7892 & 2.2340 & 2.5324 \\
\hline S50Z15C875 & 0.1455 & 0.0945 & 1.0178 & 2.2088 \\
\hline S50Z15C850 & 0.1718 & 0.1672 & 0.8753 & 1.2398 \\
\hline Put option & \multicolumn{5}{|c|}{$T=30$} \\
\hline S50Z15P950 & 0.2103 & 0.2242 & 2.0329 & 1.9923 \\
\hline S50Z15P925 & 0.3801 & 0.3901 & 3.2238 & 3.1208 \\
\hline S50Z15P900 & 0.8321 & 1.9323 & 1.3223 & 2.9023 \\
\hline S50Z15P875 & 0.3012 & 1.3223 & 2.4387 & 3.8922 \\
\hline S50Z15P850 & 0.6231 & 1.1021 & 4.2981 & 5.3892 \\
\hline Call option & \multicolumn{5}{|c|}{$T=60$} \\
\hline S50Z15C950 & 0.0133 & 0.0132 & 4.0820 & 4.0037 \\
\hline S50Z15C925 & 0.0013 & 0.3254 & 5.3244 & 5.1220 \\
\hline S50Z15C900 & 0.0023 & 0.6543 & 7.1021 & 8.3212 \\
\hline S50Z15C875 & 0.0021 & 1.1435 & 6.1213 & 7.0092 \\
\hline S50Z15C850 & 0.0032 & 1.1354 & 3.2103 & 3.9326 \\
\hline Put option & 0.1423 & 0.5424 & 8.0329 & 7.8023 \\
\hline S50Z15P950 & 0.1424 \\
\hline S50Z15P925 & 0.1124 & 0.9043 & 5.2923 & 4.2922 \\
\hline S50Z15P900 & 0.0942 & 0.4214 & 4.3232 & 5.9201 \\
\hline S50Z15P875 & 0.0032 & 1.9323 & 4.9324 & 6.2873 \\
\hline S50Z15P850 & 0.0012 & 1.5932 & 3.3200 & 4.6700 \\
\hline
\end{tabular}

From year 2016, the contracts under study are S50Z16C1000, S50Z16C975, S50Z16C950, S50Z16C925, S50Z16C900, S50Z16P1000, S50Z16P975, S50Z16P950, S50Z16P925 and S50Z16P900. Table 7 shows that the option prices from all of our randomized contracts with both times to maturity of 30 days and 60 days under GARCH model are the nearest to the option prices in the market, except only for the contract S50Z16C925 under the EGARCH model with time to maturity of 30 days that has slightly less RMSE, 0.6389, compared to the RMSE of the contract under the GARCH model, 0.6434 .

From the results it is obvious that BS model performs the highest RMSE in most of the contracts compared to GARCH, EGARCH and GJRGARCH. It is noted that, among GARCH models, all contracts under the GJRGARCH model have higher RMSE than the other contracts under the other models but lower RMSE than BS model. Also, clearly, most contracts under the GARCH model have the lowest RMSE. Although, there are few contracts that show a slightly better performance with the EGARCH model, the differences in RMSE are insignificant when compared to that of the GARCH model. As a result, it could be concluded that the GARCH model may be a more suitable model for option pricing for SET50 in Thailand, based on our sample of data.
Table 7 Model comparison for 2016 contracts.

\begin{tabular}{|c|c|c|c|c|}
\hline Contract & GARCH & EGARCH & GJR & BS \\
\hline Call option & \multicolumn{5}{|c|}{$T=30$} \\
\hline S50Z16C1000 & 0.1424 & 0.1644 & 1.0432 & 1.3020 \\
\hline S50Z16C975 & 0.1242 & 0.1453 & 1.3252 & 1.5234 \\
\hline S50Z16C950 & 0.4522 & 0.5543 & 2.4325 & 3.2992 \\
\hline S50Z16C925 & 0.6434 & 0.6389 & 1.0093 & 1.5236 \\
\hline S50Z16C900 & 0.8422 & 1.3042 & 2.9432 & 3.42321 \\
\hline Put option & \multicolumn{5}{|c|}{$T=30$} \\
\hline S50Z16P1000 & 0.4233 & 1.2325 & 3.4252 & 4.5823 \\
\hline S50Z16P975 & 0.2394 & 0.5352 & 0.4256 & 1.2832 \\
\hline S50Z16P950 & 0.3827 & 0.5623 & 3.4256 & 4.2324 \\
\hline S50Z16P925 & 0.3235 & 1.0992 & 3.9342 & 5.2312 \\
\hline S50Z16P900 & 0.1125 & 0.3453 & 4.4256 & 5.9921 \\
\hline Call option & \multicolumn{5}{|c|}{$T=60$} \\
\hline S50Z16C1000 & 0.0024 & 0.1235 & 1.2425 & 1.5422 \\
\hline S50Z16C975 & 0.0425 & 0.2352 & 2.2452 & 3.9812 \\
\hline S50Z16C950 & 0.1842 & 1.2423 & 3.2425 & 2.9902 \\
\hline S50Z16C925 & 0.0242 & 1.3523 & 1.2014 & 1.1021 \\
\hline S50Z16C900 & 0.0083 & 1.5042 & 0.3256 & 1.6202 \\
\hline Put option & \multicolumn{5}{|c|}{$T=60$} \\
\hline S50Z16P1000 & 0.0923 & 0.3425 & 2.5326 & 3.2201 \\
\hline S50Z16P975 & 0.0031 & 0.0212 & 1.4256 & 2.3324 \\
\hline S50Z16P950 & 0.0892 & 0.0993 & 3.2362 & 4.9821 \\
\hline S50Z16P925 & 0.0523 & 0.5242 & 2.2567 & 3.0583 \\
\hline S50Z16P900 & 0.1100 & 1.2154 & 3.42264 & 4.9122 \\
\hline
\end{tabular}

\section{Conclusion}

In this paper, we studied the GARCH, EGARCH and GJRGARCH models in finding the option price for the SET50 index of Thailand. We followed the method of Sui, Tong and Yang's [5] which uses the conditional Esscher transform to find the risk neutral version of the GARCH, EGARCH and GJRGARCH model which is required for finding option prices.

We carried out the exercise of simulating option prices using the GARCH, EGARCH and GJRGARCH models in the risk neutral measure $Q$. Our computations of the risk neutral version of the models are in agreement with those of Duan's [2] and Schmitt's [4].

The contracts that we studied were randomized from two years, 2015 and 2016. The option contracts from year 2015 are S50Z15C950, S50Z15C925, S50Z15C900, S50Z15C875, S50Z15C850, S50Z15P950, S50Z15P925, S50Z15P900, S50Z15P875 and S50Z15P850. The option contracts from year 2016 are S50Z16C1000, S50Z16C975, S50Z16C950, S50Z16C925, S50Z16C900, S50Z16P1000, S50Z16P975, S50Z16P950, S50Z16P925 and S50Z16P900. All of our sample option contracts are traded in the Thailand Futures Exchange (TFEX). In most of the contracts, we observed that the option prices under the GARCH model is the closest to the actual option prices in the market. Especially, when the GARCH model is compared to the well-known Black-Sholes (BS) model, GARCH model can significantly outperform BS model. Only three out of twenty contracts in this 
study, which are S50Z15C850 with time to maturity of 30 days, S50Z15C950 S50Z15C850 with time to maturity of 60 days and S50Z16C925 S50Z15C850 with time to maturity of 30 days, show that the option prices under the EGARCH model is the closest to the actual prices in the market. However, the results of these three contracts under the EGARCH model only slightly outperform the results of the GARCH model. It can be concluded that the GARCH model might be a good candidate for these three contracts as well. This implies that the GARCH option pricing model may be the most suitable tool, compared to the EGARCH and GJRGARCH models, including the well-known benchmark of BS model, for the investors to valuate the options in Thailand.

In conclusion, the advantages of this study is to indicate that option prices in Thailand under SET50 have the ARCH effect based on Ljung-Box's $Q^{2}(n)$ test. As a result, it is suggested to use GARCH models for option pricing. Our study indicates that GARCH model obviously outperform the other pricing models, including the well-known BlackSchole model because of the least RMSE in our option price samples. This illustrates the main benefit for the investor to analyze the option pricing in Thailand. The GARCH option pricing model is a good candidate as an attractive tool for model pricing in SET50 options of Thailand. The investor can confidently use GARCH model as the most important tool to valuate option prices of SET50 and to determine the status of being overpriced and underpriced of the underlying assets. Moreover, it is suggested that GARCH model can be applied to predict the option price in the future as well based on our study of data samples of SET50 in Thailand.

\section{References:}

[1] R. Costa, A. Veiga and K. Siu, "Risk Neutral Option Pricing under some special GARCH models", Thesis presented to the Postgraduate Program in Engenharia Eletrica of the Departamento de Engenharia El'etrica at Pontifical Catholic University of Rio de Janeiro, 2010.

[2] J. C. Duan, "The Garch Option Pricing Model”, Mathematical Finance, Vol.5, No.1, 1995, pp. 13-32.

[3] H. U. Gerber and E. S.W. Shiu, "Option Pricing by Esscher Transforms", Transaction of Society of Actuaries, Vol.46, 1994, pp. 99-191.

[4] C. Schmitt, "Option Pricing using EGARCH Models", ZEW Discussion Paper No. 96-20, 1996.

[5] H. Tong, T. Siu and H. Yang, "On Pricing Derivatives under GARCH Models: A Dynamic
Gerber-Shiu Approach", North American Actuarial Journal, Vol.8, No.3, 2004, pp. 1-31.

[6] H. C. Huang, Y. C. Su and W. S. Chen, "The Impact of Liquidity on GARCH Option Pricing Error during Financial Crisis", Applied Economics and Finance, Vol.4 No.4, 2017, pp. 160-168.

[7] A. Badescu, Z. Cui and J. P. Ortega, "Non-affine GARCH Option Pricing Models, VarianceDependent Kernels, and Diffusion Limits", Journal of Financial Econometrics, vol.15 no.4, 2017, pp. 602-648.

[8] Q. Hua and T. Jiang, "Option pricing based on hybrid GARCH-type models with improved ensemble empirical mode decomposition", Quantitative Finance, vol.18 no.9, 2018, pp. 1501-1515.

[9] P. Venter, E. Mare and E. Pindza, "Price discovery in the cryptocurrency option market: A univariate GARCH approach", Cogent Economics \& Finance, vol.8 no.1, 2020.

[10] P. Venter and E. Mare, "GARCH Generated Volatility Indices of Bitcoin and CRIX", Journal of Risk and Financial Management, vol.13 no.6, 2020.

[11] M. E. Anel, J. Rastegari and L. Stentoft "Option pricing with conditional GARCH models", European Journal of Operational Research, vol.289 no.1, 2021, pp. 350-363.

[12] A. Khanthavit, The inference model The pricing of index options contracts SET 50, Thammasat University Working Paper, 2007.

[13] B Tanattrin "Volatility analysis of international tourist arrival growth rates to Thailand using Garch and GJR model", Journal of Environmental Management and Tourism, vol.5 no.1, 2014, pp. 70-83.

[14] F. Black and M. Scholes, "The Pricing of Options and Corporate Liabilities", Journal of Political Economy, Vol.81, No.3, 1973, pp. 637-654.

[15] R. B. Engle, "Autoregressive conditional heteroscedasticity with estimates of the variance of United Kingdom inflation", Econometrica, Vol.50, No.4, 2004, pp. 987-1008.

[16] D. B. Nelson, "Conditional heteroscedasticity in asset returns: A new approach", Econometrica, Vol.59, No.2, 1991, pp. 347-370.

[17] S. Gokcan, "Forecasting volatility of emerging stock markets: linear versus non- linear GARCH models", Journal of forecasting, Vol.19, No.6, 2000, pp. 499-504. 


\section{Acknowledgment}

The authors would like to greatly thank the Centre of Excellence in Mathematics, CHE, Thailand, for all supports under Contract RG-01-63-01-1.

\section{Contribution of individual authors to} the creation of a scientific article (ghostwriting policy)

Both authors contributed equally to this work.

Sources of funding for research presented in a scientific article or scientific article itself

This work was financially supported by the Centre of Excellence in Mathematics, CHE, Thailand, under Contract RG-01-63-01-1.

\section{Creative Commons Attribution}

License 4.0 (Attribution $\quad 4.0$

International, CC BY 4.0)

This article is published under the terms of the Creative Commons Attribution License 4.0

https://creativecommons.org/licenses/by/4.0/deed.en US 